\title{
The 26 December 2001 Solar Event Responsible for GLE63. I. Observations of a Major Long-Duration Flare with the Siberian Solar Radio Telescope
}

\author{
V.V. Grechnev · A.A. Kochanov \\ Received ; accepted

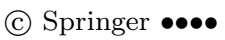

\begin{abstract}
Ground Level Enhancements (GLEs) of cosmic-ray intensity occur, on average, once a year. Due to their rareness, studying the solar sources of GLEs is especially important to approach understanding their origin. The SOL2001-12-26 eruptiveflare event responsible for GLE63 seems to be challenging in some aspects. Deficient observations limited its understanding. Analysis of extra observations found for this event provided new results shading light on the flare. This article addresses the observations of this flare with the Siberian Solar Radio Telescope (SSRT). Taking advantage of its instrumental characteristics, we analyze the detailed SSRT observations of a major long-duration flare at $5.7 \mathrm{GHz}$ without cleaning the images. The analysis confirms that the source of GLE63 was associated with an event in active region 9742 that comprised two flares. The first flare (04:30-05:03 UT) reached a GOES importance of about M1.6. Two microwave sources were observed, whose brightness temperatures at $5.7 \mathrm{GHz}$ exceeded $10 \mathrm{MK}$. The main flare, up to the M7.1 importance, started at 05:04 UT, and occurred in strong magnetic fields. The observed microwave sources reached about $250 \mathrm{MK}$. They were not static. Having appeared on the weakerfield periphery of the active region, the microwave sources moved toward each other nearly along the magnetic neutral line, approaching a stronger-field core of the active region, and then moved away from the neutral line like expanding ribbons. These motions rule out an association of the non-thermal microwave sources with a single flaring loop.
\end{abstract}

Keywords: Cosmic Rays, Solar; Flares; Radio Bursts, Microwave; Instrumentation and Data Management

Institute of Solar-Terrestrial Physics SB RAS, Lermontov St.

126A, Irkutsk 664033, Russia email: grechnev@iszf.irk.ru

email: kochanov@iszf.irk.ru 


\section{Introduction}

Solar energetic particles are somehow accelerated in association with solar eruptive events. The highest-energy particles sometimes produce in the Earth's atmosphere considerable fluxes of secondary particles, which are able to cause ground-level enhancements (GLE) of cosmic-ray intensity. GLEs are mainly detected with neutron monitors (see, e.g., Cliver, 2006; Nitta et al., 2012; and references therein).

Seventy-two GLE events caused by relativistic solar protons have been observed from 1942 to 2015. Most solar sources of the 51 GLEs registered after 1970 (Kurt et al., 2004; Cliver, 2006; Aschwanden, 2012; Gopalswamy et al., 2012, 2013; Thakur et al., 2014) were associated with major flares of the soft X-ray (SXR) GOES X class (40 GLEs, including GLE72 - see Chertok, Belov, and Grechnev, 2015) or M class (GLE28 with an M5, GLE63 with an M7.1, and GLE71 with an M5.1 flare). The GOES importance of the flare associated with GLE24 and those of the far-side sources of GLE23, GLE29, GLE39, GLE50, and GLE61 are uncertain. Atypically favorable conditions probably accounted for GLE33 (C6) and GLE35 (M1.3) associated with moderate flares, weak microwave bursts, and relatively slow coronal mass ejections (CMEs), due to shock-acceleration of a high coronal seed population (Cliver, 2006).

The rare occurrence of GLEs and their frequent association with both big flares and fast CMEs hampers identifying their origins and makes studying parent solar events highly important. The 'big flare syndrome' concept (Kahler, 1982) explained the correlation between the parameters of near-Earth proton enhancements and flare emission by a general correspondence between the energy release in an eruptive flare and its various manifestations. However, recent studies by Dierckxsens et al. (2015) and Trottet et al. (2015) indicate that both flares and shock waves can accelerate GLE particles. Grechnev et al. (2013b) revealed a scattered correlation between the peak fluxes of $>100 \mathrm{MeV}$ protons and peak flux densities of $35 \mathrm{GHz}$ bursts, although the proton outcome of four events, including GLE63 and GLE71, was much stronger. Further, Grechnev et al. (2015) found a higher correlation between the total proton and microwave fluences; nevertheless, the proton-abundant events were the outliers. Their superiority could be due to, for example, predominant shock-acceleration or contributions from stronger nearly concurrent far-side events.

The subject of the present study is the 26 December 2001 solar event with an SXR peak time at 05:40 UT (all times hereafter refer to UT) responsible for GLE63. The aim is to understand the possible causes of its atypically high proton outcome. Some other aspects of this solar event look also challenging.

The SXR emission of this event was atypically long (Aschwanden, 2012). Examination of the SXR light curves led Gopalswamy et al. (2012) to set a probable onset time of the associated flare at 05:03 UT. This time is close to the extrapolated CME onset time in the online CME catalog (http://cdaw.gsfc.nasa.gov/CME_list/ Yashiro et al., 2004) and a reported appearance of a type II burst in the range 04:59-05:02 UT. On the other hand, this burst is clearly visible at 04:53 UT and detectable still earlier in the Hiraiso Radio Spectrograph (HiRAS) spectrum (2001122605.gif) at http://sunbase.nict.go.jp/solar/denpa/hirasDB/Events/2001/ 
This slowly drifting burst evidences the presence of a moving source at least, ten minutes before the estimated onset time of a fast CME (average speed of $1446 \mathrm{~km} \mathrm{~s}^{-1}$ according to the CME catalog). Furthermore, it is not clear why the fast CME and a strong shock wave (possibly responsible for the GLE particles) developed in association with a microwave burst, which was not extremely strong. It is not also clear when and where the shock wave appeared.

The flare and eruption in this event have been studied incompletely because of limited data. The observations with the Extreme-ultraviolet Imaging Telescope (EIT: Delaboudinière et al., 1995), onboard the Solar and Heliospheric Observatory (SOHO), had a gap from 04:47 to 05:22 UT. The Transition Region and Coronal Explorer (TRACE: Handy et al., 1999) did not produce extremeultraviolet images in which an eruption could be detected. No SXR images or hard X-ray data are available.

The search for extra data revealed that the 26 December 2001 event was observed in microwaves with the Siberian Solar Radio Telescope (SSRT: Smolkov et al., 1986; Grechnev et al., 2003) at $5.7 \mathrm{GHz}$; the Nobeyama Radioheliograph (NoRH; Nakajima et al., 1994) at 17 and $34 \mathrm{GHz}$, and in $1600 \AA$ by TRACE. The analysis of extra observations found for this event has led to new results that we present in three companion papers.

This article (Paper I) addresses the SSRT observations of this flare. The SSRT routinely observes the Sun since 1996, but the difficulties to clean and calibrate the SSRT images of strong flare sources and a rather long time (typically $2-3$ min) required to produce each image restrict the opportunities to use its imaging (2D-mode) observations. The usage of the SSRT data in studies of flares has been limited (e.g. Altyntsev et al., 2002, 2007, 2016; Meshalkina et al., 2012; Alissandrakis et al.,2013). The 1D-mode observations of a higher time resolution have also been involved, especially in studies of microwave sources on short time scales.

The flare occurred near the winter solstice that is a most unfavorable season for the observations with the SSRT. At that time, its beam pattern is considerably extended in the north-south direction, and distortions associated with insufficiently accurate knowledge of instrumental characteristics reach maximum. These circumstances hamper efficient cleaning of the images. Nevertheless, a relatively low level of the SSRT beam side lobes due to a large number of antennas arranged in an equidistant array makes raw images (without cleaning) usable for an analysis.

In the present article the 26 December 2001 flare is studied from SSRT raw images as well as its $1 \mathrm{D}$ response. This has made possible to study the flare development in microwaves, to produce detailed light curves of the total flux and brightness temperature of the microwave sources at $5.7 \mathrm{GHz}$, and to analyze their spatial evolution. We focus on the opportunities provided by the SSRT data in studies of major flares and pursue the results, which can be obtained from the SSRT observations using the simplest estimations. Some of the results, which do not correspond to conventional properties of microwave flare sources, will be analyzed using observations in different spectral domains.

Analyzing the TRACE and NoRH observations along with multi-frequency total flux data, Paper II (Grechnev et al., 2016a) elaborates and clarifies the 


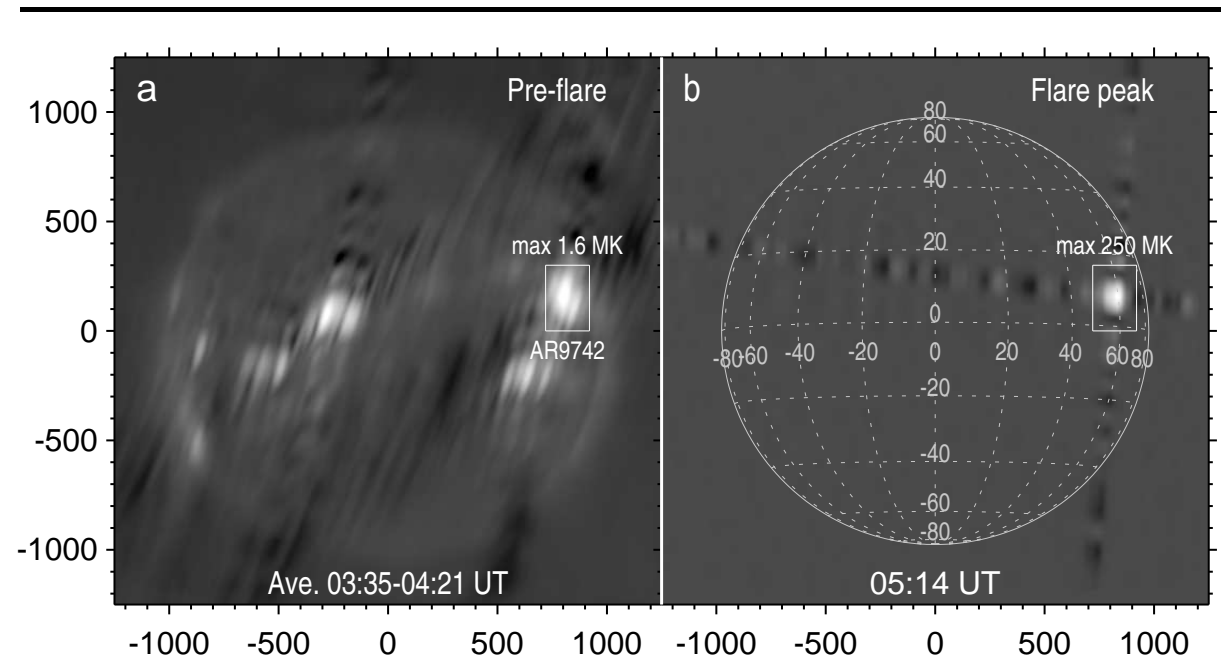

Figure 1. SSRT full-disk images (logarithmic brightness scale). (a) Averaged pre-flare image. (b) Single image at the flare peak. The maximum brightness temperatures over AR 9742, which is shown within a white rectangle, are specified in the panels. The axes indicate arc seconds from the solar disk center.

conclusions drawn in the present article. Based on the results of these two articles, Paper III (Grechnev et al., 2016b, in preparation) will address the eruption and endeavor to understand the possible causes of the high proton productivity of the 26 December 2001 solar event.

\section{Observations}

\subsection{Summary of the Flare}

The event occurred in active region (AR) 9742 (N08 W54). The AR had a $\beta \gamma$ magnetic configuration. Figure 1 a shows an average of 31 raw microwave images produced for the pre-flare interval of 03:35-04:21 UT using a recent SSRT software (Kochanov et al., 2013). Despite instrumental distortions, the average image shows both active regions and the solar disk. A moderate level of stripes is due to relatively low side lobes; the level of the first (negative) side lobes of a perfectly phase-aligned SSRT antenna array is $-22 \%$, of the second ones is $+13 \%$, etc. The side lobes from an extended source become smoothed and reduced.

The quiet-Sun brightness temperature at $5.7 \mathrm{GHz}$ is $16 \times 10^{3} \mathrm{~K}$. The brightness temperatures of the microwave sources in AR 9742 (see the white rectangle) did not exceed 1.6 MK. A single image in Figure 1b presents flare sources at the peak of the microwave burst (05:14 UT). Their brightness temperatures reached about $250 \mathrm{MK}$, so that all the other weaker sources disappeared in the image. The stripes in this image are weaker than in Figure1 1 ; they do not exceed $11 \%$ in the north-south direction and $7 \%$ in the east-west direction. This fact indicates rather large size of the sources. 


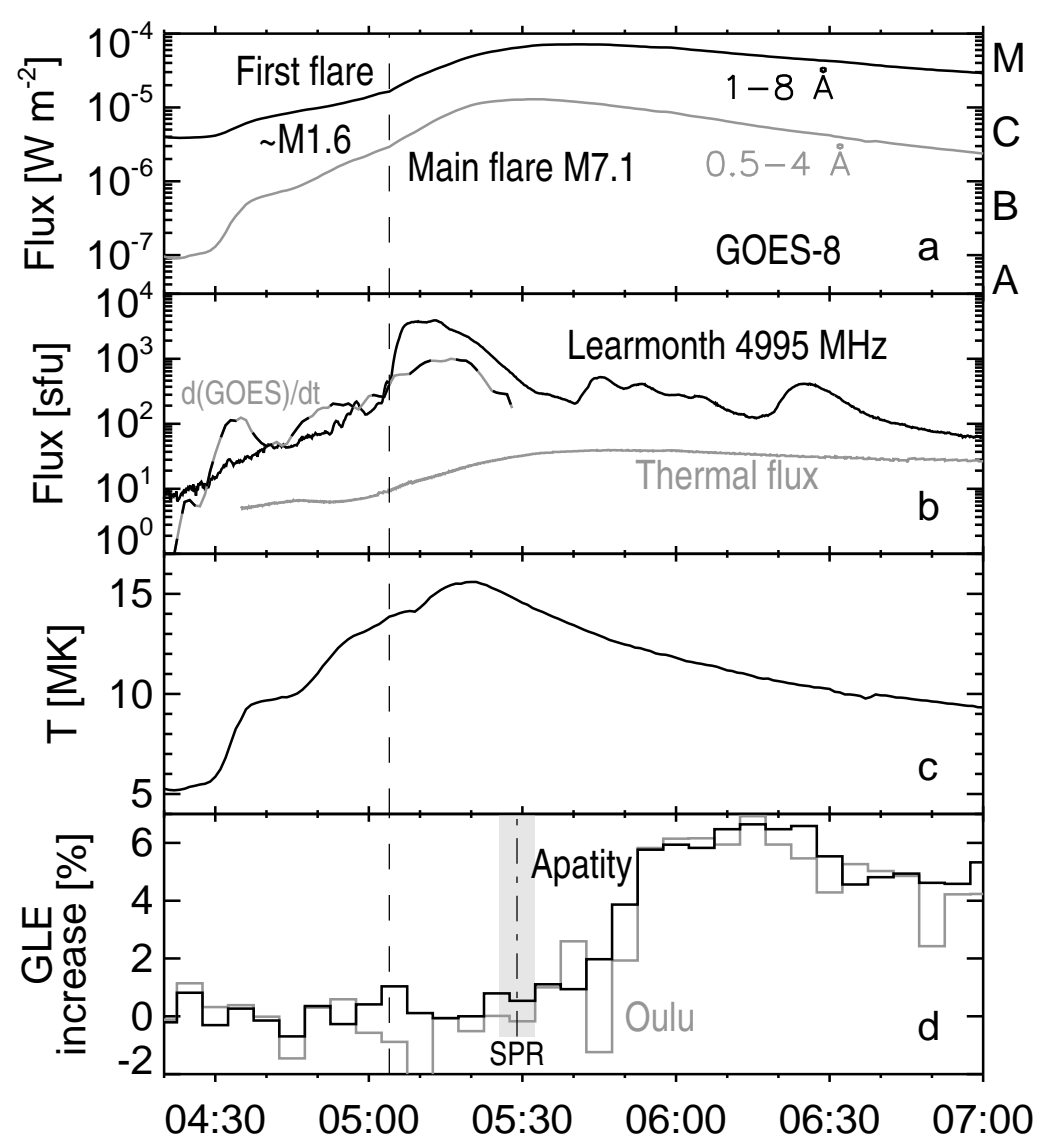

Figure 2. Light curves of the event. (a) SXR emission recorded by GOES-8. (b) Microwave burst. The gray curve represents the thermal bremsstrahlung flux estimated from GOES data. The gray curve with black dashes shows the time-derivative of the 0.5-4 Å GOES flux (positive part). (c) Temperature computed from the GOES data. (d) GLE increase recorded by the Apatity (black) and Oulu (gray) neutron monitors. The vertical dash-dotted line with shading denotes the solar particle release time with uncertainties (Reames, 2009). The vertical dashed line in all the panels separates the first flare (up to about M1.6 importance) and the main flare. All times refer to UT.

GOES-8 recorded a long-duration SXR emission starting at 04:32 UT that reached up to M7.1 at 05:40 UT (Figure 2a). The temperature calculated from the two GOES channels is shown in Figure 2r. The calculated total emission measure (not shown) reached $1.1 \times 10^{49} \mathrm{~cm}^{-3}$ at 05:03 UT (vertical dashed line) and a maximum of $4.6 \times 10^{49} \mathrm{~cm}^{-3}$ at 05:47 UT.

A microwave burst in Figure 2b was recorded in Learmonth (US Air Force Radio Solar Telescope Network) at a frequency of $4995 \mathrm{MHz}$, which is close to the observing frequency range of the SSRT. The pre-burst level was subtracted. A moderate increase of the microwave emission by 05:02 UT changed to a sharp rise up to about $4000 \mathrm{sfu}$ at 05:08-05:13 UT. The gray curve represents thermal bremsstrahlung flux calculated from the temperature and emission measure. The microwave burst was dominated by non-thermal emission. The contribution from 
thermal bremsstrahlung became significant late at the decay phase. The gray curve with black dashes presents a positive part (by 05:28 UT) of the timederivative of the $0.5-4 \AA$ flux scaled to be comparable with the microwave burst. The similarity between the shapes of the GOES derivative and the microwave burst (called the Neupert effect; Neupert, 1968) supports their common source. In summary, the SXR and microwave emissions indicate that the electron acceleration in the flare started by 04:30 UT and strengthened at 05:03 UT.

Figure $2 \mathrm{~d}$ presents the 5 -min data from the Apatity (black) and Oulu (gray) neutron monitors, which recorded GL63 up to 6.5\%. The vertical dashed-dotted line marks the solar particle release (SPR) time estimated by Reames (2009) from the velocity dispersion analysis. The shading indicates the SPR uncertainty. The delay between the flare emission and the estimated SPR time might indicate that acceleration of heavy particles was not efficient enough before 05:30 UT, or that they were accelerated simultaneously with electrons, but got an access to open magnetic fields to reach Earth after that time only. Some observational indications related to this issue will be considered in Paper III.

As mentioned, the SXR emission of the flare was atypically long. Its halfheight duration in 1-8 $\AA$ was 86 min, being twice longer than the other longest GLE-related flares of Solar Cycles 23 and 24 (44 min for GLE62, 39 min for GLE71, 37 min for GLE69, 29 min for GLE68 and GLE58; all others were still shorter). Both the rise and decay phases of the flare had very long durations. Examining the logarithmic time derivative of the SXR intensity, Gopalswamy et al. (2012) estimated the onset time of the GLE-associated flare at 05:03 UT (the vertical dashed line). From the presence of the long preceding SXR emission up to about M1.6 importance and an inflection in each of the GOES light curves at that time, Grechnev et al. (2013b) supposed the possible occurrence of a nearly concurrent stronger far-side event. It was difficult to verify this conjecture due to the lack of images, in which eruptions could be detected. Most likely, this conjecture is not confirmed; the microwave burst in Figure $2 \mathrm{~b}$ evidences an increasing non-thermal emission up to $200 \mathrm{sfu}$ at 05:02 UT, corresponding to the GOES derivative. If the only source of the microwave burst was AR 9742, then the only source of the SXR emission was the flare in this region. We will check this using the SSRT data.

\subsection{Raw SSRT Data}

The SSRT is located at geographic coordinates $\phi=\mathrm{N} 51^{\circ} 45^{\prime}, \lambda=\mathrm{E} 102^{\circ} 13^{\prime}$. From 1996 to July 2013, the SSRT operated in its initial design. At that period, including 26 December 2001, the SSRT was a cross-shaped interferometer consisting of two equidistant linear arrays, EW and NS, each of 128 antennas spaced by $d=4.9 \mathrm{~m}$. The observing frequency band was from 5.67 to $5.79 \mathrm{GHz}$ (central frequency $\nu_{\mathrm{C}}=5.73 \mathrm{GHz}$ ). Unlike modern synthesis interferometers, the SSRT was a directly-imaging telescope. Imaging was performed using the frequency dependence of the SSRT beam direction (frequency scanning). Each image of the Sun was formed in its passage through the multi-beam fan due to the diurnal rotation of the Earth (Smolkov et al., 1986; Grechnev et al., 2003). 
The signals from all antennas of each linear array came into the waveguide system, which had the structure of a binary tree, and were combined to form NS and EW output signals. The 2D correlation component was extracted using a well-known modulation technique. The phases of the output signals of the NS and EW linear interferometers were modulated and the signals were combined into in-phase and anti-phase sums. The construction existing since late 1990s did not allow extracting a response from each linear interferometer.

Due to the summation of all the antenna's signals in situ in the waveguide system, their dynamic range corresponded to the actual range of brightness temperatures of microwave sources and could be very high. For example, the dynamic range of the images in Figure 1 exceeds $2 \times 10^{4}$. The high input dynamic range is a critical point of a directly imaging telescope, thus requiring a controllable attenuator.

The receiver system of the SSRT was a spectrum analyzer. Its output presented the solar images in the time-frequency coordinates. A portion of raw SSRT data on 26 December 2001 with a main part of the microwave burst is shown in Figure 3 a (the difference of the in-phase and anti-phase signals) and in Figure $3 \mathrm{~b}$ (the sum of these signals). Each frequency (total 500 channels) corresponds to a different viewing direction. The sampling interval was $0.336 \mathrm{~s}$ in routine observations. To follow the development of the burst, Figure $3 \mathrm{~d}$ presents the variations of the maximum brightness along the dashed arc in Figure $3 \mathrm{~b}$. A way to compute this time profile is discussed later in this section.

The beam of a linear interferometer depends on a direction $\operatorname{cosine}[\cos \theta]$ with $\theta$ being a viewing direction relative to the interferometer's baseline. For the $\mathrm{EW}$ interferometer, $\cos \theta_{\mathrm{EW}}=\sin H \cos \delta$; for the NS interferometer, $\cos \theta_{\mathrm{NS}}=$ $\cos H \cos \delta \sin \phi-\sin \delta \cos \phi$, with $H$ being the hour angle and $\delta$ the declination. Each linear interferometer has major maxima in the directions where $\cos \theta_{n}=$ $n c /(\nu d)$, with numbers $n=0, \pm 1, \ldots, \pm n_{\max }, c$ the speed of light, $\nu$ a frequency, and $n$ the interference order $\left(n_{\max }=\nu d / c=93\right)$.

The portion of the SSRT observation in Figure 3 contains the local noon (near 05:12 UT). At this time $H=0, n_{\mathrm{EW}}=0$, the frequency dependence of the EW interferometer's spatial sensitivity disappears and its $1 \mathrm{D}$ response to a compact source in the time-frequency coordinates is a vertical stripe. The 1D EW responses in the other interference orders, from -14 to +7 , appear as a set of nearly vertical stripes. The 1D response of the NS interferometer appears as long arcs, symmetric relative to the local noon. Figures 3 and 3 b contain orders $n_{\mathrm{NS}}=89,90$, and 91 .

The 2D response in Figure $3 \mathrm{a}$ is formed in the passage of the Sun through successive interference orders (for each linear interferometer), which have alternate signs. The small dark and light ellipses at intersections of the interference maxima of the NS and EW interferometers are the corresponding images of the Sun. The imaging software (Kochanov et al., 2013) extracts each image with surroundings corresponding to a required field of view and transforms it to rectangular coordinates in the plane of the sky. As the burst rose, the attenuator decreased the signal (yellow plot, 18 changes in this interval). The ellipses faded relative to the burst source and disappeared right of $n_{\mathrm{EW}}=-3$, after 05:04 UT. Faint non-flaring structures started to reappear right of $n_{\mathrm{EW}}=5$, after 05:24 UT. 


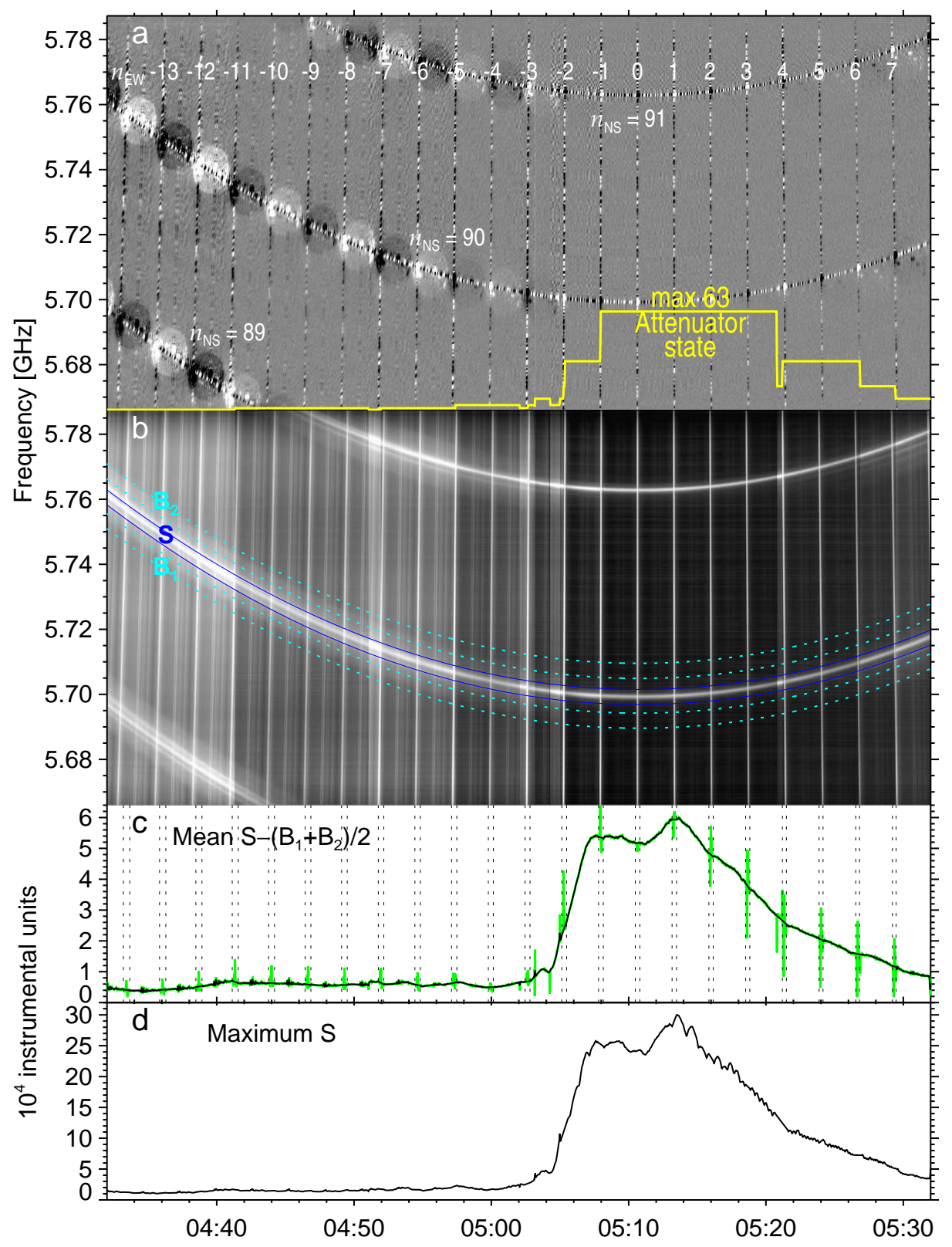

Figure 3. Raw SSRT data. (a) $2 \mathrm{D}$-mode data. The nearly vertical stripes correspond to different interference orders of the EW interferometer specified in the panel. Three long arcs correspond to interference orders 89, 90, and 91 of the NS interferometer. The yellow plot in panel (a) represents the attenuator state. (b) 1D-mode data. The long arc-like band S within the dark-blue arcs traces the NS response to the flaring source. The light-blue dotted bands $\mathrm{B}_{1}$ and $\mathrm{B}_{2}$ are used to compute the background. (c) Variations of an average over the $\mathrm{S}$ band with a subtracted averaged background. (d) Variations of the maximum over band $\mathrm{S}$. 
The 1D response in Figure 3b contains the summed additive signals from both interferometers, while the $2 \mathrm{D}$ correlation component is suppressed. The basic interference structure of the 1D data is the same as that of the 2D data. Here each sample at a given frequency represents an instantaneous total of the solar emission over a narrow stripe extracted by the EW interferometer's 1D-mode knife-edge beam summed with similar total coming from the NS interferometer.

A long bright arc in Figure 3b labeled $\mathrm{S}$ is a trace of the flare region observed by the NS interferometer in $n_{\mathrm{NS}}=90$. Appropriate corrections were introduced to compensate for the displacement of this source from the solar disk center. Portions of similar traces correspond to $n_{\mathrm{NS}}=89$ (before 04:44 UT) and $n_{\mathrm{NS}}=$ 91 (after 04:45 UT). The bright response in $n_{\mathrm{NS}}=90$ within the dark-blue arcs is a sum of the flaring microwave sources and a stripe of the quiet solar disk within the one-dimensional NS interferometer's beam.

When the flare emission became strong enough, the contribution from the quiet-Sun stripe can be neglected and the response from band $\mathrm{S}$ within the darkblue arcs reproduces approximately the total microwave flux. A corresponding plot corrected for the attenuator state changes is shown by the green line in Figure 35. The vertical broken lines delimit the intervals, in which the responses to the flare region from the EW interferometer appear. To reduce their contribution, a half-sum of the background bands, $B_{1}$ and $B_{2}$ (of the same widths as band $\mathrm{S}$ ), is subtracted. Then the light curve was smoothed with a boxcar of $11 \mathrm{~s}$ and interpolated within the intervals between the pairs of the vertical broken lines. The result is shown with the black curve.

Similarly, Figure $3 \mathrm{~d}$ shows the variations of an instant maximum over the S band. As long as the brightness distribution among the microwave flare sources is nearly constant, this plot reproduces the temporal variations of their maximum brightness temperature. A modulation with a decreasing period, that is especially clearly visible after 05:13 UT, is possibly caused by the passage of the NS interferometer's response across the sensitive cells of the spectrum analyzer. A gradual shape of this curve justifies the interpolation applied to the total-flux plot in Figure 3k. The similarity of the two plots during the major burst indicates the constancy of the total area of the microwave sources. The initial parts of the plots, where the quiet-Sun contribution is significant, are less similar.

\subsection{Calibration}

The SSRT images are calibrated referring to the quiet-Sun brightness temperature of $T_{\mathrm{QS}}=16000 \mathrm{~K}$ (Zirin, Baumert, and Hurford, 1991; Borovik, 1994). The calibration technique is based on the analysis of the brightness distribution in an image, where two statistical peaks should be present. One of them corresponds to the zero sky level and the second corresponds to the quiet-Sun level. The images are calibrated by referring to the positions of the maximum values in the two peaks (see Kochanov et al., 2013 for details). For brevity, henceforth we refer to this technique as auto-calibration. It works, as long as the microwave sources are not extremely bright, so that the quiet-Sun disk is detectable.

We have produced 105 SSRT images starting from the pre-flare stage at 03:35 UT (Figure 17) up to the late decay, by 06:28 UT. Most of them were autocalibrated, excluding the major part of the burst. To complement this interval 


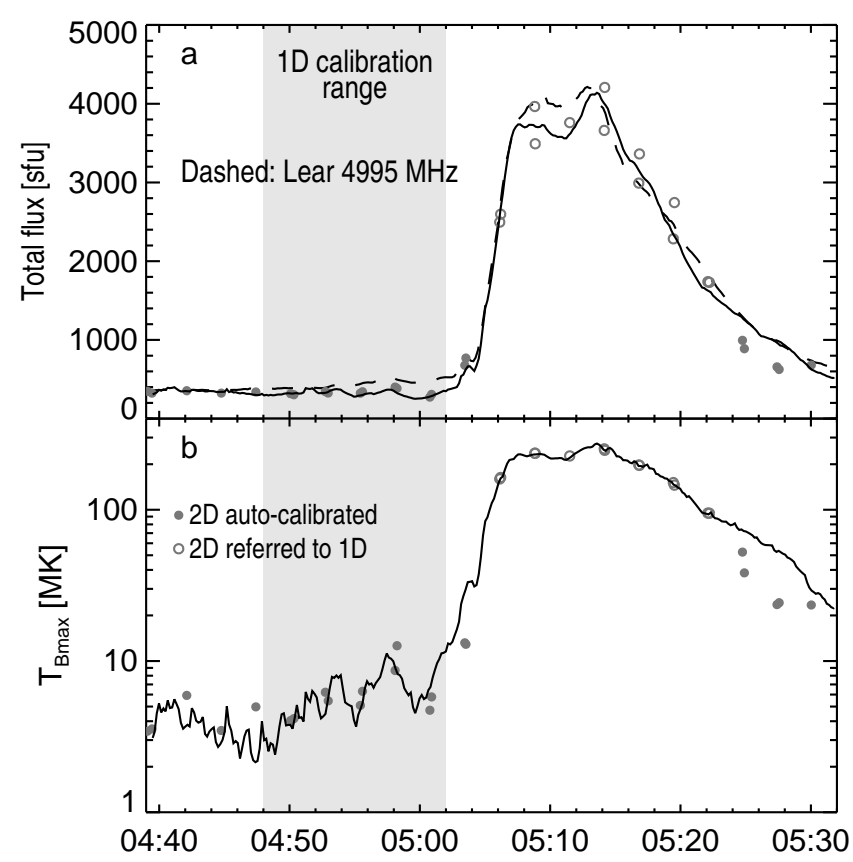

Figure 4. Time profiles of the microwave emission at $5.7 \mathrm{GHz}$ produced from SSRT data: (a) total flux and (b) maximum brightness temperature. The dashed curve in panel (a) represents the Learmonth data at $4995 \mathrm{MHz}$ for comparison. The circles represent the measurements from imaging SSRT data: filled gray circles correspond to auto-calibrated images and open circles represent the images calibrated by referring to $1 \mathrm{D}$ data. The shading denotes the interval in which $1 \mathrm{D}$ data were calibrated by referring to $2 \mathrm{D}$ images.

with calibrated SSRT data, we invoke the time profiles computed from 1D data shown in Figures 35 and 3 $\mathrm{d}$. These light curves are quantified in instrumental units. It is possible to express the time profile of the maximum brightness in absolute brightness temperatures by referring to the $2 \mathrm{D}$ images, using the rise phase of the burst, when both kinds of data are available.

Figure 4b illustrates the procedure. The maximum brightness temperatures found from auto-calibrated images are shown by the filled circles. The shading indicates the calibration interval of the 1D data. Two images around 05:03 UT were degraded by the attenuator shifts and excluded from the calibration procedure. The calibration coefficients were found by means of a linear regression. Using them, we have obtained the calibrated time profile of the maximum brightness temperature shown by the solid line. Its initial variations might not be reliable due to contributions from the other sources elsewhere on the Sun. By referring to this time profile, we calibrated the 2D images in brightness temperatures during the major burst (open circles).

When all of the 2D SSRT images have been calibrated, the total flux timeprofile can be quantified in sfu. This is shown in Figure 4a by the filled circles for auto-calibrated images and by the open circles for the images calibrated by referring to the 1D SSRT data. A relatively wide scatter is mostly due to shortcomings of the imaging software. Some of them were not revealed previously 
because of deficient experience in handling major flares. Some distortions are due to insufficiently accurate knowledge of characteristics of the SSRT systems. They are strongest when observing the Sun at low altitudes, which was the case on 26 December 2011. These causes and multiple changes of the attenuator state probably resulted in the deviations of three of the latest images.

At a final step, we have calibrated the total flux time-profile computed from 1D SSRT data by referring to the 2D images in the whole interval and using the same regression technique (solid line in Figure 4 a). To evaluate the calibration quality, the dashed line shows the total flux recorded in Learmonth at $4995 \mathrm{MHz}$. The turnover frequency of the microwave burst was close to the SSRT observation range (Grechnev et al., 2013b; Paper II); therefore, the total fluxes recorded by the SSRT at $5.7 \mathrm{GHz}$ and in Learmonth at $4995 \mathrm{MHz}$ should almost coincide. Actually, with all inaccuracies of the measurements from the 1D SSRT data, the difference between the time profiles produced from them and the Learmonth data is within $15 \%$. The scatter of the 2D SSRT data, excluding the latest problematic images, is within $20 \%$ (the previous image-to-image calibration stability reached, at best, 30\%, Grechnev et al., 2003).

The brightness temperatures at $5.7 \mathrm{GHz}$ exceeded $100 \mathrm{MK}$ during the major burst and reached $270 \mathrm{MK}$ at the maximum, stronger than the first flare by more than one order of magnitude. Adopting an uncertainty range of $\pm 15 \%$, i.e. from $230 \mathrm{MK}$ to $310 \mathrm{MK}$, we decided to choose a round value of $250 \mathrm{MK}$ (lower than the estimated $270 \mathrm{MK}$ ) within this range as a probable estimate of a highest brightness temperature reached during the burst. Such a high brightness temperature, never observed with the SSRT previously, confirms the non-thermal nature of the emission ( $c f$. Figure 2r).

Observations of major flares with the SSRT were problematic previously. It has become possible to produce the detailed microwave time profiles in this event just due to the very strong emission, which allowed neglecting a contribution from the other sources elsewhere on the Sun. Conversely, this contribution makes the approach applied here problematic in studies of weaker events.

\subsection{Microwave Sources}

\subsubsection{Main Properties}

Figure 5 shows selected microwave images of AR 9742 observed by the SSRT at $5.7 \mathrm{GHz}$ at different stages of the event. The field of view corresponds to the white frame in Figure 1. The maximum brightness temperatures over the images are specified in the panels. The white elliptic contour presents the half-height SSRT beam. The black-on-white contour traces the major magnetic polarity inversion (neutral) line at the photospheric level separating an S-polarity east region from an N-polarity west region. The neutral line was found from a longitudinal magnetogram obtained with the Michelson Doppler Imager (MDI: Scherrer et al., 1995), onboard SOHO, on 26 December at 04:51 UT.

All SSRT images show two distinct microwave sources. Their spatial structures are unresolved; thus, the real brightness temperatures were still higher. We strove to improve the coalignment accuracy between SSRT images and 


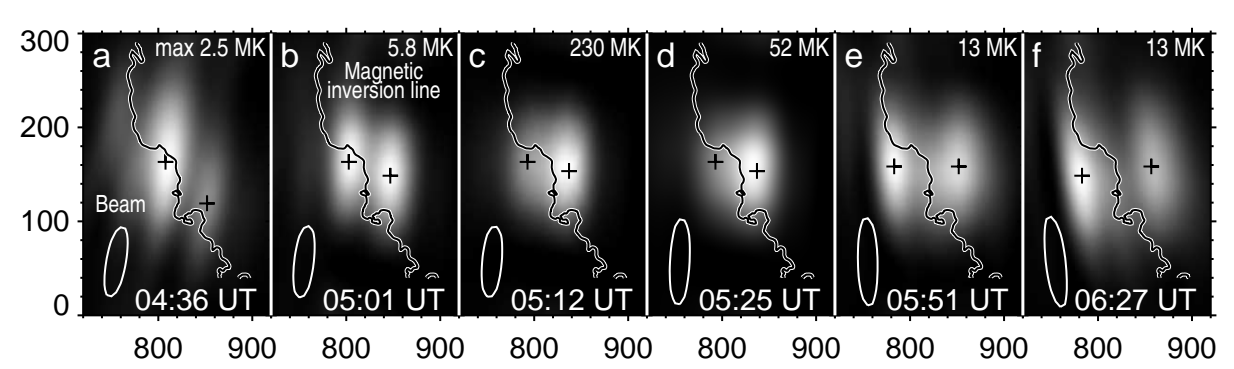

Figure 5. Microwave images of $A R 9742$ at $5.7 \mathrm{GHz}$ (linear brightness scale) observed at different stages of the event: (a) at the early onset of the first flare; (b) at its end, short before the major flare; (c) near the peak of the burst; (d) at the decay of the major burst; (e) and (f) during the late decay phase. The observation times of the images are marked by the short bars in Figure 6r. The field of view corresponds to the white frame in Figure 1 The white ellipses represent the half-height contours of the SSRT beam. The black-on-white contour represents the magnetic polarity inversion line. The crosses mark the brightness centers of the two sources.

their pointing. We found the positions of the $5.7 \mathrm{GHz}$ sources relative to the neutral line, comparing them with properly processed higher-resolution NoRH $17 \mathrm{GHz}$ images, TRACE $1600 \AA$ images, and white-light images from TRACE and SOHO/MDI. The final pointing accuracy should be within $20^{\prime \prime}$. The crosses in Figure 5 mark the brightness centers of the sources found by an automatic algorithm described in the next section.

Figure 5 a presents the onset of the first flare. The major source here is close to the neutral line, polarized by about $-7 \%$, and does not have a counterpart at $17 \mathrm{GHz}$. These properties are similar to those of the sources associated to the neutral line, which are typically related to the origin of strong flares (see, e.g., Uralov et al., 2008). A somewhat increased brightness temperature of this source of $2.5 \mathrm{MK}$ can be due to an additional gyrosynchrotron emission from the flare, which had already started. Another, weaker source $(1.4 \mathrm{MK})$ is located west of the neutral line. A faint extended feature in the upper-left corner is due to a beam side lobe.

The centers of the sources in the other images were located at different sides of the neutral line. Both sources were polarized. In Figures 5 - 5 ; , the centers of the sources move toward each other along the neutral line. The distance between them decreases. Around the flare peak, the sources overlapped (Figures 5: and 5d), so that the distance between their centers could be somewhat underestimated at that time. Then, the centers moved away from the neutral line in Figures $5 \mathrm{~d}-5 \mathrm{f}$. The angle between the line connecting the centers of the sources and the neutral line increased. A weaker diffuse brightening appeared between the major sources in the late decay phase (Figures 55 and [5), possibly due to thermal bremsstrahlung from the upper part of the flare arcade.

The relation between the brightness temperatures of the two sources was not constant. The source associated to the neutral line was brighter before the flare. The ratio of the maximum brightness temperatures of the west to east source initially was in the range $0.5-0.6$, gradually increased to $1.0-1.1$ during the first flare, reached 1.5-1.7 during the main flare, and then decreased to $0.7-$ 1.1. While the maximum brightness temperatures changed during the event by 
two orders of magnitude, a narrow range of the variations in the ratio between the two sources indicates a similarity of their time profiles.

Comparison of the images in Figure 5 with half-height contours of the SSRT beam indicates that the sources were not compact. Decomposition of the eastwest and north-south cross-sections of the image observed near the peak of the burst in Figure 55. has resulted in an observed size of the sources of $\approx 43^{\prime \prime} \times 94^{\prime \prime}$, and a deconvolved size of $\approx 38^{\prime \prime} \times 59^{\prime \prime}$ (with a beam of $21^{\prime \prime} \times 73^{\prime \prime}$ at that time). A maximum brightness temperature of the deconvolved strongest west source at that time should approximately be proportional to the ratio between the observed area and the deconvolved one, i.e., $230 \times 1.82 \approx 420 \mathrm{MK}$.

\subsubsection{Motions of the Microwave Sources}

To measure the distances between the observed sources and their orientation relative to the neutral line, we used a model elliptic source of $43^{\prime \prime} \times 60^{\prime \prime}$ extended in the north-south direction. The convolution of this source with an idealized SSRT beam (the real beam is not known precisely) looks more or less similar to the observed sources.

The algorithm measuring the positions of the sources operated in the following way. At the first step, a starting estimate was found for the position of the maximum over each image. A model response (computed as the convolution of the model source with the SSRT beam) was scaled to the maximum, placed into this position, and subtracted from the image. The position of the brightness center was calculated for the second source. Then, a model response corresponding to the second source was subtracted and a final estimate for the position of the first source was calculated. This procedure supplied the arrays of the $(x, y)$ coordinates for the two sources measured from 105 images. To reduce the scatter in the measurements, both the $x$ and $y$ arrays of each source were filtered using the median over three neighbors and smoothed with a boxcar average over five neighbors. The parameters of the model source somehow affect the results, but do not change them significantly.

Figures $6 \mathrm{a}$ and $6 \mathrm{~b}$ present the detailed measurements of the relative positions and shear angle of the two sources in a wide time interval. The angle is referred to a major orientation of the neutral line of $105^{\circ}$ from the west direction, without considering its detailed curves. The relative measurements do not depend on the accuracy, with which the images were coaligned with each other and with the magnetogram. To relate the motions of the sources to the development of the flare, Figure 6r. presents the total flux time-profile of the burst at $4995 \mathrm{MHz}$ recorded at Learmonth. This time profile shows various features in detail without a scatter, which is present in the time profiles computed from the 2D SSRT data.

Along with the measured distances between the brightness centers of the two sources, Figure 6h presents the full width at half-maximum (FWHM) of a model response in the east-west direction, where it was the narrowest. The half-height width of a response puts the limit to resolve two features. If the two sources are located too close, then the results can be biased. The measurements within the shaded interval, when the sources overlap in the images, should therefore be considered with caution. 


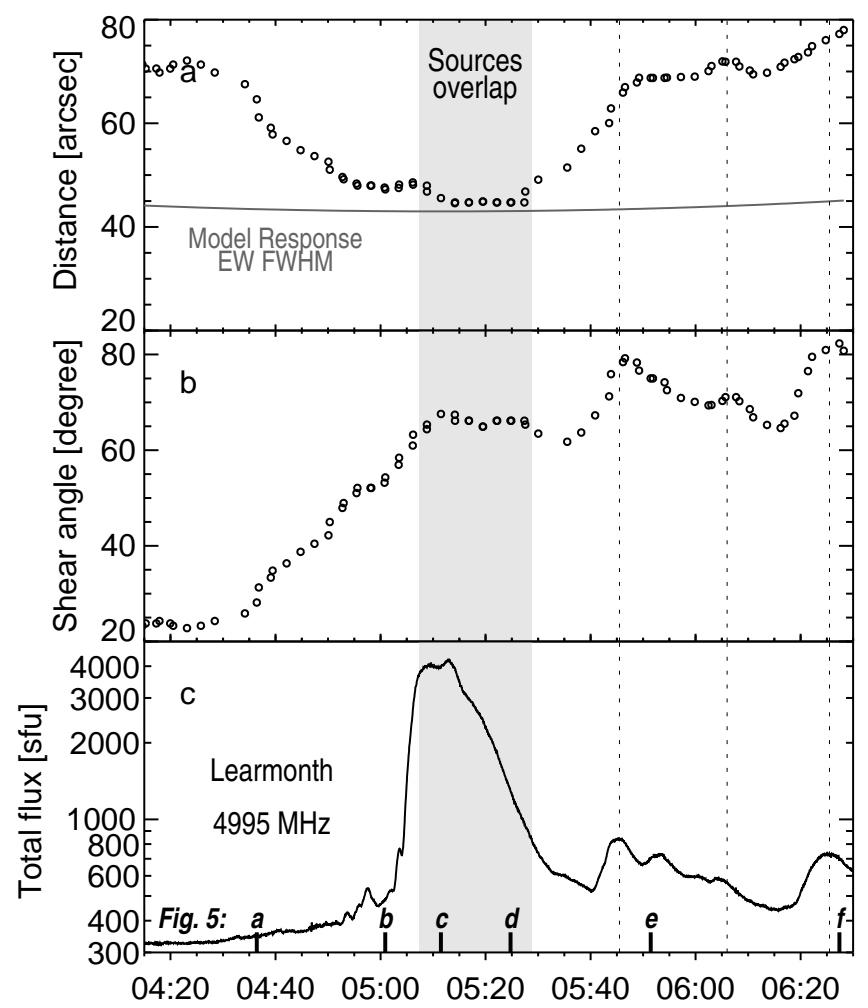

Figure 6. Relative motions of the microwave sources observed at $5.7 \mathrm{GHz}$. (a) The distance between the brightness centers of the two sources. The gray line represents a half-height width of a model response in the east - west direction. The shading marks the interval when the two sources overlap in the images. (b) The shear angle between the line connecting the brightness centers of the two sources and the magnetic neutral line. (c) The total flux time-profile of the burst recorded at $4995 \mathrm{MHz}$ in Learmonth (including pre-burst emission). The vertical dotted lines mark some minor features in the time profile. The short thick bars with labels at the bottom denote the observation times of the images in the corresponding panels of Figure 5

The measured positions seem to be affected by subsidiary bursts, whose sources might be located away from the brightness centers of the major burst. These minor features in the time profile in Figure 6r marked with the vertical dotted lines have detectable counterparts in Figure $6 \mathrm{~b}$, and some in Figure $6 \mathrm{a}$.

The overall evolution corresponds to what can be deduced from the images in Figure 5. The distance between the sources in Figure 6a gradually decreased, starting at the onset of the first flare, reached a minimum during the major burst, and then gradually increased. The shear angle between the positions of the microwave sources and the neutral line in Figure $6 \mathrm{~b}$ increased, starting at the onset of the first flare, and after the major burst became non-monotonic, most likely, due to subsidiary bursts. 


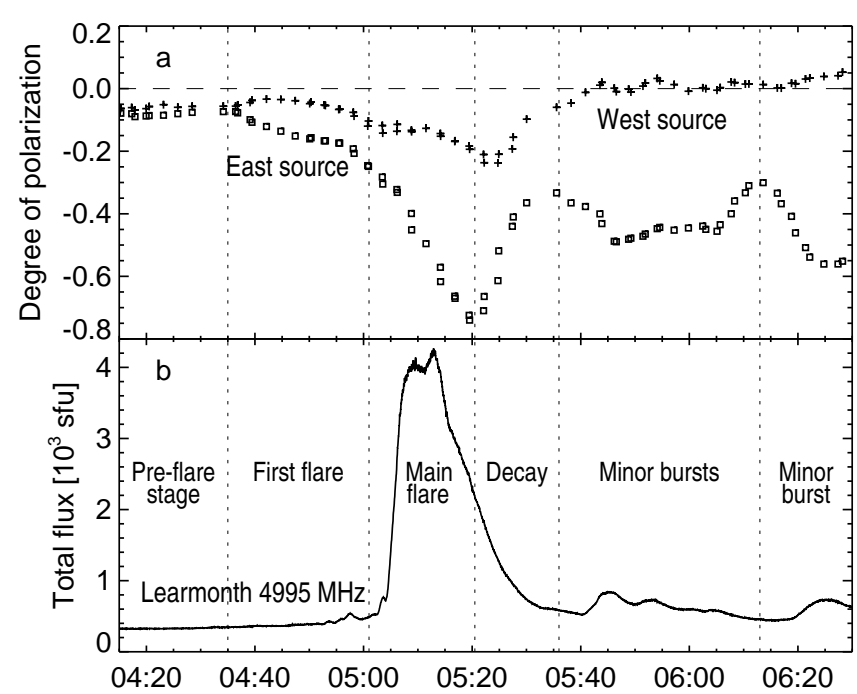

Figure 7. Variations of the degree of polarization in the central regions of the east (squares) and west (crosses) microwave sources (a) in comparison with a total flux time profile (b). The vertical dotted lines separate different episodes of the event indicated in panel (b).

\subsubsection{Degree of Polarization}

Despite the complexity of the polarization structure, the measured positions of the brightness centers for the two sources allow studying how the degree of polarization evolved. Its value was measured for each source as an average within a small area surrounding its brightness center. This area was defined by a contour of the SSRT beam at a level of 0.65 . The results are practically insensitive to the changes of the level from 0.5 to 0.8 , which we tested. The measurements are related to the whole observed configuration without subtraction of the quasistationary background. To reduce the scatter, the data points were smoothed with a boxcar average over five neighbors. The degree of polarization calculated in this way does not depend on the accuracy of the coalignment or calibration. The contributions from side lobes, edge effects, and other instrumental issues are minimized thereby. The results are presented in Figure $7 \mathrm{a}$ in comparison with the total flux time-profile in Figure $7 \mathrm{~b}$.

The time profiles in Figure 7 a show complex variations of the polarization with a general correspondence to the main stages of the event. This correspondence along with a gradual character of the variations supports the reliability of the measurements from each image. The parameters of the model source used to measure the positions of the brightness centers somehow affect the results, but do not change them significantly.

Before the flare onset, the degree of polarization was around $-7 \%$ in both sources. The polarization of the east source started to gradually strengthen with the onset of the first flare, reached $-23 \%$ at its end, and, during the main flare, strengthened steeply. During the peak of the burst it increased from $-45 \%$ to $-60 \%$, and reached $-75 \%$, when the flux of the burst decreased to a half-height 
level. During the subsequent decay, the polarization of the east source weakened. Its later variations were apparently related to subsidiary bursts.

The polarization of the west source was inverted and was considerably weaker. Its variations from 04:50 to 05:36 UT were similar to those of the east source. The maximum degree of polarization was about $-20 \%$ soon after the strongest polarization of the east source. During the minor bursts after 05:36 UT, the variations of the polarization in the two sources became dissimilar.

\section{Discussion and Summary}

Both SXR and microwave data show that the long-duration flare in AR 9742 consisted of two parts. The first flare started at about 04:30 UT, lasted half an hour, and reached a GOES importance of about M1.6 at 05:03 UT. The main flare, which was much stronger in microwaves, started at 05:04 UT and reached an importance of M7.1 at 05:40 UT. The major microwave burst fully developed and mainly finished within 36 min, before the SXR peak. The decay of the SXR emission possibly lengthened due to subsidiary bursts during 05:40-06:10 UT and around 06:25 UT. The SXR emission corresponded to the microwave burst via the Neupert effect. The only source of the microwave burst was AR 9742, and the only source of the SXR emission was the flare in this region. Most likely, the solar event in AR 9742 associated with the M7.1 flare was the only source of the near-Earth proton event and GLE63.

In the preceding studies of microwave flare emissions, the sources observed by the SSRT at $5.7 \mathrm{GHz}$ were typically associated with loop-top regions (e.g. Altyntsev et al., 2002, 2007, 2016; Meshalkina et al.,2012, and others). In the 26 December 2001 flare, two distinct microwave sources were observed. They were polarized, comparable in size and brightness temperatures (up to extremely high values), and varied rather similarly. These properties of the two sources and their positions, most likely at different sides of the magnetic neutral line, indicate their association to the conjugate legs of a closed magnetic structure.

Observations of flares in thermal emissions ( $\mathrm{H} \alpha$, ultraviolet, extreme ultraviolet, soft X-rays, etc.) typically show complex multi-loop structures. By contrast, microwave and hard X-ray (HXR) images of non-thermal emissions in impulsive flares usually reveal simpler configurations identified with one or two loops (e.g., Hanaoka, 1996, 1997; Nishio et al., 1997; Grechnev and Nakajima, 2002; and many others). Moreover, an analysis of the microwave morphologies in many near-the-limb events led Tzatzakis, Nindos, and Alissandrakis (2008) to a conclusion about single-loop microwave configurations existing even in some long-duration major flares. These observational indications resulted in a prevailing concept of a single microwave-emitting flare loop (or, at most, two loops). However, even the inhomogeneous flare-loop model, initially proposed by Alissandrakis and Preka-Papadema (1984) and further developed using powerful modeling tools to account for several inhomogeneities (Tzatzakis, Nindos, and Alissandrakis, 2008; Kuznetsov, Nita, and Fleishman, 2011), cannot explain various observations. In particular, Zimovets, Kuznetsov, and Struminsky (2013) demonstrated that, at least, some of the seemingly single loops shown 
by microwave NoRH images corresponded to multi-loop arcades observed with telescopes of a higher spatial resolution.

The concept of a single microwave-emitting flare loop is difficult to reconcile with systematic motions of the microwave sources in Figure [6a. A continuous relative displacement of the two sources by $\gtrsim 20^{\prime \prime}$ in the plane of the sky implies their association with different loops or loop systems at different times. In principle, the observed relative motions of the sources could be caused by a varying height of the emitting regions in two legs of a single loop. This effect should also be manifested in the degree of polarization due to a varying magnetic field strength and viewing angle. However, while the variations in the relative distance in Figure 6a before 05:20 UT are nearly symmetric to those after 05:20 UT, the shear angle in Figure [6 and polarization in Figure $7 \mathrm{~b}$ are strongly asymmetric. Thus, while the height variations are possible, the observed motions were most likely determined by the displacements of the footpoint regions of numerous arcade loops.

Similar motions are known from HXR observations. From a statistical study of HXR sources, Bogachev et al. (2005) found different types of their motions relative to the magnetic neutral line and interpreted them in terms of the standard flare model.

The diverging motion at the decay phase (05:30-05:45 UT and later) resembles a usual expansion of the flare ribbons, which represent the footpoint regions of numerous loops. This is type I motion according to Bogachev et al. (2005). The approach of the two sources toward each other along the neutral line throughout the first flare is also difficult to relate to a single loop; this kind of motion was also observed in HXR (type II motion in Bogachev et al., 2005). The deviations from the systematic motions during subsidiary bursts also imply more complex configurations than a single microwave-emitting flare loop.

Additional indications are provided by the degree of polarization. This parameter of the gyrosynchrotron emission is closely related to the magnetic field strength, being not directly dependent on an unknown number of emitting electrons. Keeping this in mind, the varying degree of polarization in Figure $7 \mathrm{~b}$ is difficult to understand in terms of the single-loop hypothesis. On the other hand, with the limited data we consider, a probable participation of additional loops makes the interpretation of these complex variations ambiguous. We therefore consider, for simplicity, the approximation of a single homogeneous source for each of the two observed microwave sources.

To analyze the gyrosynchrotron emission, analytic approximations by Dulk and Marsh (1982) and Dulk (1985) are widely used. The accuracy of the formulas is reduced at low harmonics of the gyrofrequency; in such situations, we invoke them to obtain rough estimates and to understand major tendencies, and additionally refer to the plots in those articles that present the results of numerical calculations. Note that gyroresonance features in the spectrum are not expected in observations due to inhomogeneities of the magnetic field.

The polarization of the east source corresponded to the $x$-mode emission and reached $r_{\mathrm{c}} \approx-0.75$. This is only possible, if the optical thickness $[\tau]$ was small $(\tau<1)$. According to Dulk (1985), the degree of circular polarization, $r_{\mathrm{c}}$, in the 
optically thin limit is

$$
r_{\mathrm{c}} \approx 1.26 \times 10^{0.035 \delta} \times 10^{-0.071 \cos \theta}\left(\frac{\nu}{\nu_{B}}\right)^{-0.782+0.545 \cos \theta} \quad\left(\tau_{\nu} \ll 1\right),
$$

with $\delta$ being a power-law index of the number density spectrum of microwaveemitting electrons, $\theta$ a viewing angle, and $\nu_{B} \approx 2.8 \times 10^{6} B$ the electron gyrofrequency in the magnetic field $[B]$. The degree of polarization directly depends on the magnetic field strength.

The polarization of the east source strengthened until the decay phase. During the first flare, this occurred presumably due to an increasing contribution of the gyrosynchrotron emission, which became dominant at the end of the first flare (Figure 2b); and, possibly, due to the motion of the source from a weakerfield periphery of the active region to its stronger-field core. With a degree of polarization of $-(0.20-0.25)$ just before the main flare and a viewing angle around $\theta \approx 60^{\circ}$, corresponding to the position of AR 9742, the magnetic field strength in the east source should not exceed $-100 \mathrm{G}$. This conclusion applies to the whole first flare.

An increasing degree of the polarization during the major burst suggests a strengthened magnetic field in the east source. Assuming for certainty $\theta=60^{\circ}$ and $\delta=2.5-3.5$ (see Paper II), with $r_{\mathrm{c}} \approx-0.5$ at the peak of the burst, we estimate the magnetic field strength in the east source to be around $-250 \mathrm{G}$ at that time. With $r_{\mathrm{c}} \approx-0.75$ at the end of the major burst, the magnetic field strength in the coronal east source could reach $\approx-540 \mathrm{G}$. This magnetic field corresponds to $\nu / \nu_{B} \approx 4$, beyond the validity range of the Dulk (1985) approximation, but nevertheless consistent with his Figure 3. If the optical thickness of this source was not small enough to satisfy the condition $\tau_{5.7} \ll 1$, then the magnetic field should be somewhat stronger.

The magnetic field at the photosphere underneath should be considerably stronger than in the low corona. The magnetic field in this region of the MDI magnetogram on that day at 04:51 UT ranged from -600 to $-850 \mathrm{G}$. Since AR 9742 was not far from the limb, this magnetogram might be strongly affected by the projection effect. We additionally examined an MDI magnetogram observed two days before, at 04:51 UT on 24 December. The magnetic fields at about this place reached more than $-1000 \mathrm{G}$; on the other hand, the active region evolved. Thus, the estimated maximum magnetic field strength of $\approx-540 \mathrm{G}$ in the east coronal source seems to be plausible.

The west source was polarized in the sense of the $o$-mode emission, with a degree, not exceeding $-20 \%$. Either its intrinsic emission corresponded to the $x$-mode and was inverted, propagating through a layer of the quasi-transversal magnetic field, or it was initially optically thick. If the SSRT observing frequency, $5.7 \mathrm{GHz}$, was higher than the peak frequency of this source, then its brightness temperature should depend on the magnetic field strength directly (Dulk and Marsh, 1982; Dulk, 1985). However, a weak magnetic field of $-(40-45) \mathrm{G}$ corresponding to $r_{\mathrm{c}}=-0.2$ with the same $\delta=2.5-3.5$ and $\theta=60^{\circ}$ would contradict a higher brightness temperature of the west source relative to the east source. Hence, the west source was not optically thin. Its peak frequency, 
$\nu_{\text {peak }}$, was either slightly lower than $5.7 \mathrm{GHz}$ (inverted $x$-mode emission), or, most likely, higher (intrinsic o-mode emission). The latter option is consistent with an estimated $\nu_{\text {peak }} \approx 6.9 \mathrm{GHz}$ for the total flux in this event (Grechnev et al., 2013b; see also Paper II). Indeed, the total flux is the sum of the emissions from the east source with a $\nu_{\text {peak }}<5.7 \mathrm{GHz}$, and the west source, whose peak frequency should be $>5.7 \mathrm{GHz}$, even if the total flux had $\nu_{\text {peak }} \geq 5.7 \mathrm{GHz}$.

The peak frequency can be estimated, referring again to Dulk and Marsh (1982) and Dulk (1985), as

$$
\nu_{\text {peak }} \approx 2.72 \times 10^{3} \times 10^{0.27 \delta}(\sin \theta)^{0.41+0.03 \delta}(N L)^{0.32-0.03 \delta} \times B^{0.68+0.03 \delta},
$$

where $(N L)$ is a column density of emitting electrons. Although it can be different in the two sources located in the conjugate legs of the same closed structure, the dependence of $\nu_{\text {peak }} \propto(N L)^{0.22-0.25}$ is considerably weaker than $\nu_{\text {peak }} \propto B^{0.76-0.79}$. While the basic formula might be inaccurate at a low harmonic of the gyrofrequency, a stronger magnetic field seems nevertheless to be a most probable reason for a higher $\nu_{\text {peak }}$ in the west source.

A brightness temperature of $\approx 4.2 \times 10^{8} \mathrm{~K}$ estimated in Section 2.4 .1 for the deconvolved west source near the peak of the burst (Figure 5k) roughly corresponds, with $\delta=2.5-3.5$ and $\theta=60^{\circ}$, to the optically thick emission around the fifth harmonic (Figures 3 in Dulk and Marsh, 1982 and Dulk, 1985), i.e., $400 \mathrm{G}$ vs. $\approx 250 \mathrm{G}$ in the east source at the same time. All of the estimates, along with a behavior of the polarization during the main flare, indicate that the west source was optically thick and located in a stronger, relative to the east source, magnetic field, which increased in the course of the flare. This conclusion is supported by a higher brightness of the west source throughout the event at both 17 and $34 \mathrm{GHz}$ in the NoRH movie, norh20011226_0505_pfi.mpeg, available at http://solar.nro.nao.ac.jp/norh/html/event// entry 20011226_0505. Both sources were optically thin at these two frequencies, and therefore their brightness temperatures directly depended on the magnetic field strength.

The magnetogram observed on 26 December shows the photospheric magnetic fields in the west part of AR 9742 to be around $1000 \mathrm{G}$. A sunspot was there. A nearly radial magnetic field in its central part should be substantially reduced in the line-of-sight magnetogram observed close to the limb. We have not radialized the magnetogram to avoid overestimating the magnetic field in the region under the east source. The MDI magnetogram on 24 December shows the magnetic fields exceeding $2700 \mathrm{G}$ in the central part of the sunspot. Strong magnetic fields were really present on the photosphere approximately under the west source, which was the brightest during the main flare.

Some of the estimated magnetic field strengths fall outside the range where the accuracy of the formulas by Dulk and Marsh (1982) and Dulk (1985) is guaranteed. Nevertheless, our results are supported by the following facts. i) Our estimates are also consistent with the results of numerical calculations by Dulk and Marsh (1982) and Dulk (1985) in their Figures 3. ii) Comparison of the positions of the two sources in Figures $5 \mathrm{~b}-5 \mathrm{~b} \mathrm{~d}$ with the magnetogram confirms that magnetic fields under the west source were stronger than those under the east source, as considered in this section, while their probable values in the 
corona correspond to the estimates. iii) The NoRH movie of the flare observed at 17 and $34 \mathrm{GHz}$ also supports our results.

We conclude that the main flare occurred in strong magnetic fields, whose photospheric base, most likely, had a strength of $\gtrsim 1000$ G. Probably, the west flare ribbon extended into the strongest magnetic fields above the sunspot. However, the spatial resolution of the SSRT and the coalignment accuracy are insufficient to judge to what extent this occurred. This issue will be addressed in Paper II, which will also analyze the microwave spectrum.

As mentioned in Section 1, the onset time of the main flare corresponds to an estimated launch time of the CME. The major phase of the 26 December 2001 GLE63-related event resembles those of the 20 January 2005 event (GLE69; Grechnev et al., 2008) and of the 13 December 2006 event (GLE70; Grechnev et al., 2013a). Furthermore, Grechnev et al. (2013b) showed flaring in stronger magnetic fields above the sunspot umbrae to be typical of big proton events.

Like the previously mentioned events, the flare on 26 December 2001 involved rather strong magnetic fields and occurred, at least, close to a sunspot. The magnetic fields involved in the GLE63-related flare were probably not so strong as in the flares related to GLE69 and GLE70, when the peak frequencies exceeded $25 \mathrm{GHz}$, and the fluxes at $35 \mathrm{GHz}$ were considerably higher than $10^{4}$ sfu. Nevertheless, major aspects of these events look qualitatively similar.

An additional particularity of the 26 December 2001 event was the very long duration of the flare. The rise phase of the main flare alone lasted 36 min vs. $18 \mathrm{~min}$ for the flares related to GLE69 and GLE70. According to the Neupert effect (Neupert, 1968), this phase corresponds to the effective particle acceleration in a flare. A considerably higher correlation between the fluences of near-Earth proton enhancements, on the one hand, and fluences of the SXR and microwave emissions, on the other hand found by Grechnev et al. (2015) indicates a dependence of the total number of high-energy protons arriving at the Earth orbit on both the intensity and total duration of the acceleration process. The role of the flare duration is obvious, if protons are accelerated simultaneously with electrons in a flare, but it is more difficult to expect such a correspondence, if protons are accelerated by shock waves far away from a flare region. Therefore, a considerably higher correlation between the fluences of protons and flare emissions than between their peak values found for the 26 December 2001 event indicates a significant contribution from flare processes to the acceleration of protons. The discussion of the particle event will be further addressed in Paper III.

\subsection{Conclusion}

Our analysis has confirmed that the solar event in active region 9742 associated with the M7.1 flare was the only source of the near-Earth proton event and GLE63. No signs of a concurrent far-side event have been found.

The event in AR 9742 consisted of two parts. The first flare (04:30 - 05:03 UT) reached a GOES importance of about M1.6. The brightness temperatures at $5.7 \mathrm{GHz}$ exceeded $10 \mathrm{MK}$. The main flare, up to M7.1 importance, started at 05:04 UT, when a CME was launched. The microwave sources reached about 
250 MK. The SSRT data indicate that strong magnetic fields were involved in the main flare. These magnetic fields were probably associated with the sunspot in the west part of AR 9742.

The two microwave sources observed at $5.7 \mathrm{GHz}$ initially approached each other along the magnetic neutral line and then moved away from it like expanding ribbons. These motions are difficult to understand in the frame of a single-loop hypothesis. A natural explanation of the observed properties of the microwave sources might be their association to the legs of the flare arcade. To verify this conjecture, microwave data should be compared with the flare arcade or ribbons observed in a different spectral range, where they are clearly visible. These issues will be addressed in Paper II. The possible causes of the high proton productivity of the 26 December 2001 event will be considered in Paper III.

This is a first detailed study of a major long-duration flare from combined $2 \mathrm{D}$ and 1D SSRT data. A relatively low side-lobe level of the SSRT beam and rather large areas of the microwave sources allowed using the images produced by the SSRT without cleaning. The techniques described here provide an opportunity to study important major flares recorded with the SSRT in the past. The analysis has revealed shortcomings of the imaging and calibration software that were not manifested previously because of a deficient experience in handling major flares. Some imperfect techniques and software (e.g., calibration routines) have been improved in the course of our study. The development of some others is in progress.

The SSRT routinely carried out imaging observations of the whole Sun at $5.7 \mathrm{GHz}$ based on the initial operating principle from 1996 to July 2013. Currently, the central part of the antenna array is under reconstruction to upgrade the SSRT to the multi-frequency $(4-8 \mathrm{GHz})$ Siberian Radio Heliograph (SRH: Lesovoi et al., 2012, 2014). The remaining part of the original antenna array keeps on the initial-principle observations.

Acknowledgments We thank A.T. Altyntsev for the idea of this study and useful remarks, and our colleagues for their contribution, efforts, and assistance. The data used here are provided by the SSRT team in Badary. S.A. Anfinogentov has substantially contributed to the collaborative development of the SSRT raw-data processing and calibrating software and assisted in computations. S.V. Lesovoy developed the data acquisition system and the routine imaging software. We thank him and A.M. Uralov for fruitful discussions. We appreciate the memories of T.A. Treskov, one of the major developers of the SSRT, whose ideas helped us to implement the techniques described here, and N.N. Kardapolova, who managed the SSRT observations for many years. We thank the reviewer for useful remarks.

We are grateful to the instrumental teams of SOHO/MDI (ESA and NASA), GOES, USAF RSTN Network, and Nobeyama Radioheliograph.

A.K. was supported by the Russian Foundation of Basic Research under grants 15-32-20504 mol-a-ved and 15-02-01089.

Disclosure of Potential Conflicts of Interest The authors declare that they have no conflicts of interest.

\section{References}

Alissandrakis, C.E., Preka-Papadema, P.: 1984, Astron. Astrophys. 139, 507. 
Alissandrakis, C.E., Kochanov, A.A., Patsourakos, S., Altyntsev, A.T., Lesovoi, S.V., Lesovoya, N.N.: 2013, Publ. Astron. Soc. Japan 65, SP1, S8. DOI: 10.1093/pasj/65.sp1.S8

Altyntsev, A.T., Grechnev, V.V., Meshalkina, N.S., Yan, Y.: 2007, Solar Phys. 242, 111. DOI: $10.1007 / \mathrm{s} 11207-007-0207-9$

Altyntsev, A., Meshalkina, N., Mészárosová, H., Karlický, M., Palshin, V., Lesovoi, S.: 2016, Solar Phys. 291, 445. DOI: 10.1007/s11207-016-0846-9

Altyntsev, A.T., Sych, R.A., Grechnev, V.V., Meshalkina, N.S., Rudenko, G.V.: 2002, Solar Phys. 206, 155. DOI: 10.1023/A:1014989213205

Aschwanden, M.J.: 2012, Space Sci. Rev. 173, 3. DOI: 10.1007/s11214-011-9865-x

Bogachev, S.A., Somov, B.V., Kosugi, T., Sakao, T.: 2005, Astrophys. J. 630, 561. DOI: $10.1086 / 431918$

Borovik, V.N.: 1994, Adv. Solar Phys. 432, 185. DOI: 10.1007/3-540-58041-7_217

Chertok, I.M., Belov, A.V., Grechnev, V.V.: 2015, Solar Phys. 290, 1947. DOI: $10.1007 / \mathrm{s} 11207-015-0738-4$

Cliver, E.W.: 2006, Astrophys. J. 639, 1206. DOI: 10.1086/499765

Delaboudinière, J.-P., Artzner, G.E., Brunaud, J., Gabriel, A.H., Hochedez, J.F., Millier, F., Song, X.Y., Au, B., Dere, K.P., Howard, R.A., et al.: 1995, Solar Phys. 162, 291. DOI: $10.1007 /$ BF00733432

Dierckxsens, M., Tziotziou, K., Dalla, S., Patsou, I., Marsh, M.S., Crosby, N.B., Malandraki, O., Tsiropoula, G.: 2015, Solar Phys. 290, 841. DOI: 10.1007/s11207-014-0641-4

Dulk, G.A.: 1985, Annu. Rev. Astron. Astrophys. 23, 169. DOI: 10.1146/annurev.aa.23.090185.001125

Dulk, G.A., Marsh, K.A.: 1982, Astrophys. J. 259, 350. DOI: 10.1086/160171

Gopalswamy, N., Xie, H., Yashiro, S., Akiyama, S., Mäkelä, P., Usoskin, I.G.: 2012, Space Sci. Rev. 171, 23. DOI: 10.1007/s11214-012-9890-4

Gopalswamy, N., Xie, H., Akiyama, S., Yashiro, S., Usoskin, I.G., Davila, J.M.: 2013, Astrophys. J. Lett. 765, L30. DOI: 10.1088/2041-8205/765/2/L30

Grechnev, V.V., Nakajima, H.: 2002, Astrophys. J. 566, 539. DOI: 10.1086/338053

Grechnev, V.V., Lesovoi, S.V., Smolkov, G.Y., Krissinel, B.B., Zandanov, V.G., Altyntsev, A.T., Kardapolova, N.N., Sergeev, R.Y., Uralov, A.M., Maksimov, V.P., Lubyshev, B.I.: 2003, Solar Phys. 216, 239. DOI: 10.1023/A:1026153410061

Grechnev, V.V., Kurt, V.G., Chertok, I.M., Uralov, A.M., Nakajima, H., Altyntsev, A.T., Belov, A.V., Yushkov, B.Y., Kuznetsov, S.N., Kashapova, L.K., Meshalkina, N.S., Prestage, N.P.: 2008, Solar Phys. 252, 149. DOI: 10.1007/s11207-008-9245-1

Grechnev, V.V., Kiselev, V.I., Uralov, A.M., Meshalkina, N.S., Kochanov, A.A.: 2013a, Publ. Astron. Soc. Japan 65, SP1, S9. DOI: 10.1093/pasj/65.sp1.S9

Grechnev, V.V., Meshalkina, N.S., Chertok, I.M., Kiselev, V.I.: 2013b, Publ. Astron. Soc. Japan 65, SP1, S4. DOI: 10.1093/pasj/65.sp1.S4

Grechnev, V.V., Kiselev, V.I., Meshalkina, N.S., Chertok, I.M.: 2015, Solar Phys. 290, 2827. DOI: $10.1007 / \mathrm{s} 11207-015-0797-6$

Grechnev, V.V., Uralov, A.M., Kiselev, V.I., Kochanov, A.A.: 2016, Solar Phys. submitted (Paper II).

Hanaoka, Y.: 1996, Solar Phys. 165, 275. DOI: 10.1007/BF00149715

Hanaoka, Y.: 1997, Solar Phys. 173, 319. DOI: 10.1023/A:1004953003558

Handy, B.N., Acton, L.W., Kankelborg, C.C., Wolfson, C.J., Akin, D.J., Bruner, M.E., Caravalho, R., Catura, R.C., Chevalier, R., Duncan, D.W., et al.: 1999, Solar Phys. 187, 229. DOI: $10.1023 / \mathrm{A}: 1005166902804$

Kahler, S.W.: 1982, J. Geophys. Res. 87, 3439. DOI: 10.1029/JA087iA05p03439

Kochanov, A., Anfinogentov, S., Prosovetsky, D., Rudenko, G., Grechnev, V.: 2013, Publ. Astron. Soc. Japan 65, SP1, S19. DOI: 10.1093/pasj/65.sp1.S19

Kurt, V., Belov, A., Mavromichalaki, H., Gerontidou M.: 2004, Ann. Geophys. 22, 2255. DOI: 10.5194/angeo-22-2255-2004

Kuznetsov, A.A., Nita, G.M., Fleishman, G.D.: 2011, Astrophys. J. 742, 87. DOI: $10.1088 / 0004-637 \mathrm{X} / 742 / 2 / 87$

Lesovoi, S.V., Altyntsev, A.T., Ivanov, E.F., Gubin, A.V.: 2012, Solar Phys. 280, 651. DOI: $10.1007 / \mathrm{s} 11207-012-0008-7$

Lesovoi, S.V., Altyntsev, A.T., Ivanov, E.F., Gubin, A.V.: 2014, Res. Astron. Astrophys. 14, article id. 864-868. DOI: 10.1088/1674-4527/14/7/008

Meshalkina, N.S., Altyntsev, A.T., Zhdanov, D.A., Lesovoi, S.V., Kochanov, A.A., Yan, Y.H., Tan, C.M.: 2012, Solar Phys. 280, 537. DOI: 10.1007/s11207-012-0065-y 
Nakajima, H., Nishio, M., Enome, S., Shibasaki, K., Takano, T., Hanaoka, Y., Torii, C., Sekiguchi, H. et al.: 1994, Proc. IEEE 82, 705. DOI: 10.1109/5.284737

Neupert W.M.: 1968, Astrophys. J. Lett. 153, L59. DOI: 10.1086/180220

Nishio, M., Yaji, K., Kosugi, T., Nakajima, H., Sakurai, T.: 1997, Astrophys. J. 489, 976. DOI: $10.1086 / 304793$

Nitta, N.V., Liu, Y., DeRosa, M.L., Nightingale, R.W.: 2012, Space Sci. Rev. 171, 61. DOI: $10.1007 / \mathrm{s} 11214-012-9877-1$

Reames, D.V.: 2009, Astrophys. J. 693, 812. DOI: 10.1088/0004-637X/693/1/812

Scherrer, P.H., Bogart, R.S., Bush, R.I., Hoeksema, J.T., Kosovichev, A.G., Schou, J., Rosenberg, W., Springer, L., Tarbell, T.D., Title, A., et al.: 1995, Solar Phys. 162, 129. DOI: 10.1007/BF00733429

Smolkov, G.I., Pistolkors, A.A., Treskov, T.A., Krissinel, B.B., Putilov, V.A.: 1986, Astrophys. Spa. Sci. 119, 1. DOI: 10.1007/BF00648801

Thakur, N., Gopalswamy, N., Xie, H., Mäkelä, P., Yashiro, S., Akiyama, S., Davila, J.M.: 2014, Astrophys. J. Lett. 790, L13. DOI: 10.1088/2041-8205/790/1/L13

Trottet, G., Samwel, S., Klein, K.-L., Dudok de Wit, T., Miteva, R.: 2015, Solar Phys. 290, 819. DOI: $10.1007 / \mathrm{s} 11207-014-0628-1$

Tzatzakis, V., Nindos, A., Alissandrakis, C.E.: 2008, Solar Phys. 253, 79. DOI: 10.1007/s11207-008-9263-z

Uralov, A.M., Grechnev, V.V., Rudenko, G.V., Rudenko, I.G., Nakajima, H.: 2008, Solar Phys. 249, 315. DOI: 10.1007/s11207-008-9183-y

Yashiro, S., Gopalswamy, N., Michalek, G., St. Cyr, O.C., Plunkett, S.P., Rich, N.B., Howard, R.A.: 2004, J. Geophys. Res. 109, A07105. DOI: 10.1029/2003JA010282

Zimovets, I.V., Kuznetsov, S.A., Struminsky, A.B.: 2013, Astron. Lett. 39, 267. DOI: 10.1134/S1063773713040063

Zirin, H., Baumert, B.M., Hurford, G.J.: 1991, Astrophys. J. 370, 779. DOI: 10.1086/169861 